\title{
Successful intracardiac electrical conversion of atrial flutter in patients with complex congenital heart disease
}

David Cunningham, Jane Somerville, John A Kennedy, Edward Rowland, Anthony F Rickards

\begin{abstract}
Sixteen patients presenting on 21 occasions with atrial flutter in association with complex congenital heart disease were treated by intracardiac stimulation techniques combined with activation mapping. Nineteen episodes of atrial flutter were successfully converted to sinus rhythm. In the remaining two episodes atrial fibrillation was induced with spontaneous conversion to sinus rhythm within 12 hours in one episode and immediate DC cardioversion to sinus rhythm in the other. Intracardiac stimulation techniques were highly successful in this group and allowed reliable conversion to sinus rhythm without general anaesthesia and high energy cardioversion. In patients with atrial flutter associated with congenital heart disease intracardiac stimulation techniques should be tried first.
\end{abstract}

Atrial flutter is common after surgical repair of complex congenital cardiac malformations and it can have profound haemodynamic consequences, particularly when ventricular function is impaired. Both drug treatment and external cardioversion with high energy DC shock have disadvantages. ${ }^{1-3}$

Pharmacological agents can either allow the

Department of

Biomedical

Engineering, Royal

Brompton National

Heart and Lung

Hospital, London

D Cunningham

Paediatric and

Adolescent Unit,

Royal Brompton

National Heart and

Lung Hospital, London

J Somerville

Cardiac Department,

Western Infirmary,

Glasgow

J A Kennedy

Department of

Pacing and

Electrophysiology,

Royal Brompton

National Heart and

Lung Hospital, London

E Rowland

A F Rickards

Correspondence to

Dr David Cunningham,

Royal Brompton Nationa

Heart and Lung Hospital,

Sydney Street,

London SW $36 \mathrm{NP}$

Accepted for publication

12 February 1991 ventricular response to be controlled or may achieve chemical conversion to sinus rhythm. The conversion rate is low in these patients with congenital heart disease and residual lesions, especially when flutter has been present for a long time.

Even when the ventricular rate is controlled the circulation still lacks the atrial contribution to cardiac output and this may predispose the patients to worsening of atrioventricular valve regurgitation, particularly in hearts with one functioning ventricle, deteriorating right ventricular function in transposition of the great arteries, or in tetralogy of Fallot with pulmonary regurgitation. In general, the symptoms get worse when atrial flutter becomes established.

External DC cardioversion is effective in restoring sinus rhythm but it requires a general anaesthetic and the ventricular myocardium is liable to be damaged if repeated cardioversions are necessary. So it may be better to avoid cardioversion in a young patient with an abnormal ventricle. An alternative approach is to use intracardiac stimula- tion techniques to restore sinus rhythm. Rapid overdrive pacing ${ }^{4-7}$ and long duration single pulses ("atrioversion") ${ }^{8}$ have both been successful.

We examined the results of these techniques in a group of young patients presenting with atrial flutter in association with a complex congenital cardiac malformation and deteriorating clinical state.

\section{Patients and methods} METHODS

Patients were brought to the electrophysiological laboratory unsedated and in the post-absorptive state. Routine premedication for electrophysiological studies was used. The patients were routinely anticoagulated at the time of study. In most the study was performed as an elective procedure after the patient had been anticoagulated with oral anticoagulants for 4-6 weeks. In these patients warfarin was stopped for 24 hours before the study or until the International Normalised Ratio fell below 2 . In those in whom the study was performed as an urgent procedure half dose heparinisation was used at the start of the catheterisation.

Under local anaesthesia we introduced one or more quadripolar electrode catheters (with 5 or $10 \mathrm{~mm}$ interelectrode spacing) from a femoral or subclavian vein and advanced the catheter(s) to the right atrium. In three episodes the catheter entered the left atrium because of an atrial septal defect or absent interatrial septum.

A surface electrocardiogram and intracardiac electrograms were recorded on a multichannel inkjet recorder. Endocardial stimulation was performed by manipulating the catheter(s) into various atrial sites. Four surface electrocardiographic leads were simultaneously recorded. Atrial sites were classified as high, mid, or low and as anterior, lateral, posterior, or septal in the right atrium. Left atrial stimulation was not performed.

Through one pair of electrodes we delivered stimuli with a pulse width of $2 \mathrm{~ms}$ at twice diastolic threshold. Single stimuli were introduced after variable programmed delays from the previous spontaneous flutter electrogram to scan the atrial flutter cycle. The initial delay was $10 \mathrm{~ms}$ less than the flutter cycle length; this was reduced until refractoriness was encountered. The duration of the poststimulation (return) flutter cycle was noted. A non-compensatory return cycle resulted in the flutter being reset (type I response (fig 1)). A 
Figure 1 An example of reset (type I) and compensatory (type II) responses to atrial stimulation during flutter. (A) Three surface and three intracardiac leads ( two from mid-anterior right atrium (MARA) and low lateral right atrium (LLRA)). Flutter cycle length is $230 \mathrm{~ms}$. $A$ premature stimulus at the low lateral site produced a cycle of $180 \mathrm{~ms}$, followed by a non-compensatory return cycle of $230 \mathrm{~ms}$ ( $\mathrm{a}$ type I response). ( $B$ ) Four surface leads and one intracardiac lead from mid-posterior right atrium (MPRA). Flutter cycle length was $215 \mathrm{~ms}$. $A$ premature stimulus resulted in a cycle of 175 ms followed by $a$ compensatory return cycle of $255 \mathrm{~ms}(175+255=$ $2 \times 215$ ) (a type II response).

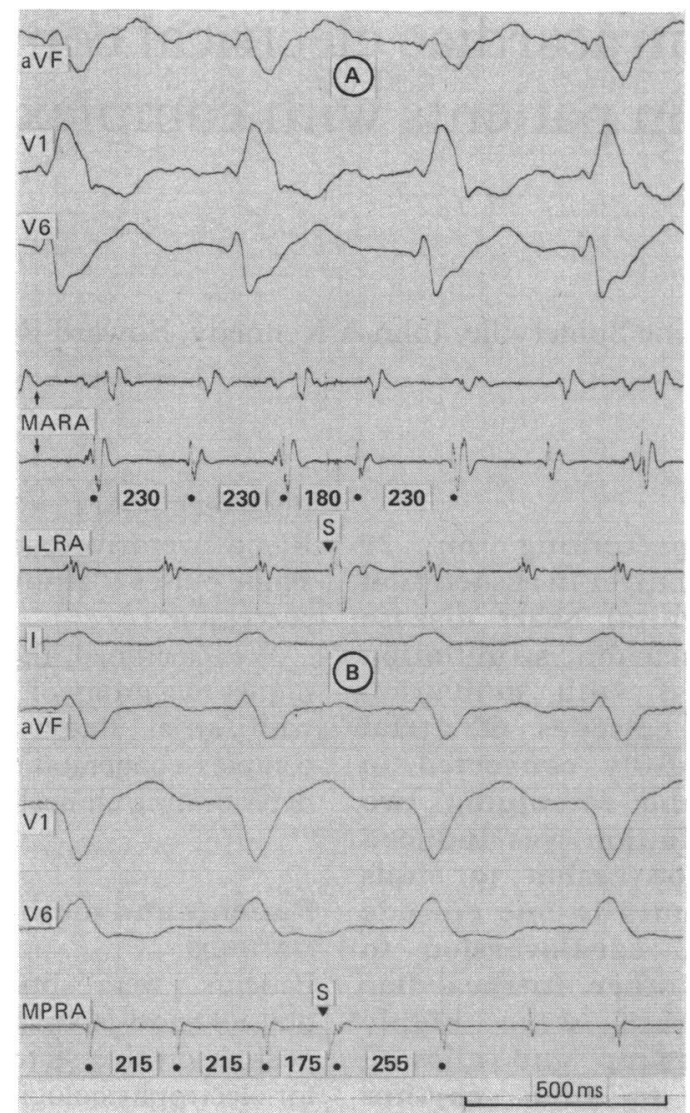

compensatory pause in the return cycle did not result in resetting (type II response). Further programmed stimuli (up to a total of four) were added if a type I response was found. Each additional stimulus was initially added at a coupling time $10 \mathrm{~ms}$ less than the flutter cycle length and reduced until refractoriness was encountered or conversion achieved.

If conversion was not achieved rapid atrial overdrive pacing was attempted with a modification of the protocol suggested by Waldo et al. $^{7}$ Pacing was begun 10 beats per minute faster than the flutter rate, initially for two seconds then if necessary for a further burst duration of four, eight, 15, or 30 seconds. If flutter persisted, the pacing rate was increased by 10 beats per minute and the sequence of burst durations was repeated. It is known that pacing rates that exceed $135 \%$ of the flutter rate are more likely to result in atrial fibrillation, ${ }^{8}$ and these rates were not exceeded unless pacing had failed at lower rates at more than one site.

If conversion was not achieved a further type I atrial site was sought and the full stimulation protocol was repeated. If a suitable type I site could not be found the full conversion protocol was carried out at a type II site.

In one patient (two episodes) a new technique (Atrioversion ${ }^{8}$ ) was used instead of rapid atrial pacing, as part of an experimental protocol. A single constant current pulse with a duration $20 \mathrm{~ms}$ longer than the flutter cycle length was delivered $10 \mathrm{~ms}$ after the refractory period (as defined by a single stimulus) with an amplitude that was initially twice the diastolic threshold and was doubled to a maximum of 20 $\mathrm{mA}$.
ELECTROCARDIOGRAPHIC DEFINITION OF ATRIAL FLUTTER

The diagnosis of atrial flutter was made from the surface electrocardiogram. The diagnosis required the presence of regular, rapid atrial activity with some degree of atrioventricular block, either spontaneously or during carotid sinus massage. An atrial rate of $>200$ beats per minute was required with clear evidence of classic flutter waves in at least one surface electrocardiographic lead. This rate is lower than the generally accepted lower rate limit for atrial flutter (240-250 beats per minute) because previous cardiac surgery and congenital abnormalities of the atrium can slow the flutter rate.

In episodes in which the atrial rate was below 200 beats per minute flutter was diagnosed only if the patient was receiving antiarrhythmic drug treatment likely to slow the atrial rate and if an earlier electrocardiogram had showed flutter at a rate greater than 200 with an identical flutter wave configuration before drug treatment.

Atrial flutter was classified as being of the "common" form if negative sawtooth deflections were seen in the inferior surface electrocardiogram leads and uncommon if the flutter waves were positive in leads I and II.

\section{PATIENTS}

Twenty one procedures were carried out on 16 patients (eight male, and eight female, aged 1543 years, mean 26). Table 1 shows the clinical details. All patients had had previous corrective surgery for their congenital lesions on at least one and up to four occasions. Eleven patients had one procedure and five had two on separate hospital admissions. The duration of flutter before attempted conversion ranged from six hours to five years (median 14 days). At the time of study, the atrial rate was $158-363$ beats per minute (mean 229) and the ventricular rate was 63-172 beats per minute (mean 100). 2:1 atrioventricular block was present in seven episodes, 3:1 block in four episodes, 1:1 conduction in one episode, and a variable block in the remainder.

The current antiarrhythmic drug treatment was amiodarone in seven episodes, digoxin in two episodes, digoxin and quinidine in two episodes, digoxin and disopyramide in two episodes, quinidine in one episode, amiodarone and digoxin in one episode, digoxin and flecainide in one episode, and xamoterol in one episode. In four episodes the patient was receiving no antiarrhythmic drug treatment at the time of study.

In five episodes the atrial rate was below 200 beats per minute. In four of these the patient was receiving amiodarone and had previous evidence of atrial flutter with an atrial rate of over 240 beats per minute before amiodarone and an identical flutter wave configuration. In the remaining episode, the flutter rate was 188 when the patient was receiving disopyramide and it accelerated to 330 in a subsequent episode when the patient's treatment was changed to xamoterol.

Conversion of atrial flutter was attempted only during episodes in which there was 
Table 1 Data on patients

\begin{tabular}{|c|c|c|c|c|c|c|c|c|}
\hline Case & Age & Sex & Basic diagnosis & Previous surgery & Residual lesions & Previous arrhythmias & Previous treatment & $\begin{array}{l}\text { Result of conversion } \\
\text { and follow up }\end{array}$ \\
\hline 1 & 17 & $\mathbf{M}$ & $\begin{array}{l}\text { VSD, infundibular } \\
\text { pulmonary } \\
\text { stenosis }\end{array}$ & $\begin{array}{l}\text { VSD closure and } \\
\text { resection of } \\
\text { pulmonary stenosis } \\
\text { (age 14) }\end{array}$ & $\begin{array}{l}\text { Very small VSD, } \\
\text { mild pulmonary } \\
\text { stenosis }\end{array}$ & $\begin{array}{l}\text { AFl + syncope } \\
\text { (age 17) } \\
\text { (requiring } \\
\text { cardioversion) }\end{array}$ & $\begin{array}{l}\text { Quinidine then } \\
\text { amiodarone }\end{array}$ & $\begin{array}{l}\text { Sinus rhythm, } \\
\text { flutter recurred } \\
\text { age } 20 \text {, repeat } \\
\text { conversion to } \\
\text { sinus rhythm }\end{array}$ \\
\hline 2 & 33 & $\mathbf{F}$ & $\begin{array}{l}\text { ASD (ostium } \\
\text { secundum) }\end{array}$ & ASD repair (age 33) & & $\begin{array}{l}\text { SVT + cerebral } \\
\text { embolism (age } \\
\text { 32) }\end{array}$ & Nil & Sinus rhythm \\
\hline 3 & 20 & $\mathrm{~F}$ & $\begin{array}{l}\text { ASD (ostium } \\
\text { secundum) }\end{array}$ & ASD repair (age 5) & & $\begin{array}{l}\text { Persistent atrial } \\
\text { flutter since age } 5\end{array}$ & $\begin{array}{l}\text { Digoxin }+ \\
\text { amiodarone }\end{array}$ & $\begin{array}{l}\text { Sinus rhythm- } \\
\text { reverted to atrial } \\
\text { flutter } 1 \text { month; } \\
\text { controlled by } \\
\text { digoxin }\end{array}$ \\
\hline 4 & 28 & $\mathbf{M}$ & Tetralogy of Fallot & $\begin{array}{l}\text { L Blalock total repair } \\
\text { (age 9) }\end{array}$ & $\begin{array}{l}\text { Small VSD, } \\
\text { pulmonary } \\
\text { regurgitation, } \\
\text { pulmonary } \\
\text { stenosis, } \\
\text { cardiomegaly }\end{array}$ & $\begin{array}{l}\text { Atrial tachycardia } \\
\text { (age 26), atrial } \\
\text { flutter (age 28) }\end{array}$ & $\begin{array}{l}\text { Amiodarone, } \\
\text { digoxin, } \\
\text { disopyramide }\end{array}$ & $\begin{array}{l}\text { Sinus rhythm, } \\
\text { flutter recurred } \\
\text { age } 31 \text {, repeat } \\
\text { conversion to SR }\end{array}$ \\
\hline 5 & 31 & $M$ & $\begin{array}{l}\text { VSD, infundibular } \\
\text { stenosis, } \\
\text { pulmonary } \\
\text { hypertension }\end{array}$ & $\begin{array}{l}\text { VSD closure } \\
\text { infundibular } \\
\text { resection (age 11) }\end{array}$ & $\begin{array}{l}\text { Pulmonary } \\
\text { hypertension, } \\
\text { right heart } \\
\text { failure }\end{array}$ & $\begin{array}{l}\text { Atrial flutter (age } \\
\text { 31) }\end{array}$ & Digoxin & $\begin{array}{l}\text { Sinus rhythm, } \\
\text { heart/lung } \\
\text { transplant age } 32\end{array}$ \\
\hline 6 & 31 & $\mathbf{F}$ & $\begin{array}{l}\text { Tricuspid atresia, } \\
\text { ASD }\end{array}$ & $\begin{array}{l}\text { Pott's operation (age } \\
18 \text { months) }\end{array}$ & $\begin{array}{l}\text { Tricuspid atresia, } \\
\text { ASD }\end{array}$ & $\begin{array}{l}\text { Atrial fibrillation } \\
\text { (age 27), } \\
\text { complete heart } \\
\text { block (age 30) } \\
\text { (pacemaker } \\
\text { insertion) }\end{array}$ & $\begin{array}{c}\text { Amiodarone, } \\
\text { digoxin }\end{array}$ & Sinus rhythm \\
\hline 7 & 34 & $\mathbf{M}$ & $\begin{array}{l}\text { Tetralogy of Fallot, } \\
\text { ASD, anomalous } \\
\text { origin of LAD }\end{array}$ & $\begin{array}{l}\text { Pott's operation (age } \\
\text { 3) and total repair } \\
\text { + ASD closure } \\
\text { (age 14) }\end{array}$ & $\begin{array}{l}\text { Pulmonary } \\
\text { regurgitation, } \\
\text { pulmonary } \\
\text { stenosis, PA } \\
\text { stenosis, } \\
\text { anomalous origin } \\
\text { LAD }\end{array}$ & $\begin{array}{l}\text { Syncope age } 30, \\
\text { ventricular } \\
\text { extrasystoles }\end{array}$ & Digoxin & $\begin{array}{l}\text { Sinus rhythm, } \\
\text { flutter recurred } \\
\text { age } 35 \text {, repeat } \\
\text { conversion to } \\
\text { sinus rhythm }\end{array}$ \\
\hline 8 & 22 & $\mathbf{F}$ & $\begin{array}{l}\text { Tricuspid atresia, } \\
\text { ASD, Fontan at } \\
\text { age } 12\end{array}$ & $\begin{array}{l}\text { Glenn's operation } \\
\text { (age } 8 \text { months), L } \\
\text { Blalock (age 8), } \\
\text { Fontan (age 12), } \\
\text { redo Fontan (age } \\
\text { 22) }\end{array}$ & & $\begin{array}{l}\text { VT (age 19), } \\
\text { ?SVT/VT (age } \\
\text { 20) }\end{array}$ & $\begin{array}{l}\text { Disopyramide, } \\
\text { amiodarone }\end{array}$ & $\begin{array}{l}\text { Sinus rhythm, } \\
\text { flutter recurred } \\
\text { age } 23 \text {, repeat } \\
\text { conversion to } \\
\text { sinus rhythm }\end{array}$ \\
\hline 9 & 22 & $\mathbf{F}$ & $\begin{array}{l}\text { TGA, VSD, } \\
\text { pulmonary } \\
\text { hypertension }\end{array}$ & $\begin{array}{l}\text { Blalock-Hanlon (age } \\
2 \text { months), } \\
\text { banding of PA (age } \\
18 \text { months), } \\
\text { palliative Mustard } \\
\text { (age } 8 \text { ) }\end{array}$ & $\begin{array}{l}\text { Pulmonary } \\
\text { hypertension, } \\
\text { VSD, tricuspid } \\
\text { regurgitation }\end{array}$ & SVT (age 17) & Amiodarone & Sinus rhythm \\
\hline 10 & 15 & $\mathbf{M}$ & $\begin{array}{l}\text { Mitral atresia, } \\
\text { doublet outlet } \\
\text { LV, ASD }\end{array}$ & Blalock-Hanlon & & & Amiodarone & Sinus rhythm \\
\hline 11 & 19 & $\mathbf{F}$ & $\begin{array}{l}\text { PDA, ostium } \\
\text { primum ASD, } \\
\text { coarctation }\end{array}$ & $\begin{array}{l}\text { PDA ligated (age } 10 \\
\text { months), ASD } \\
\text { repaired (age 5) }\end{array}$ & $\begin{array}{l}\text { Coarctation of } \\
\text { aorta }\end{array}$ & $\begin{array}{l}\text { AVNRT/SN } \\
\text { disease (age 11), } \\
\text { antitachycardia } \\
\text { pacemaker } \\
\text { removed age } 17 \\
\text { (infection) }\end{array}$ & Digoxin & $\begin{array}{l}\text { Atrial fibrillation, } \\
\text { immediately } \\
\text { converted to } \\
\text { sinus with } 75 \mathrm{~J} \\
\text { DC }\end{array}$ \\
\hline $\begin{array}{l}12 \\
13\end{array}$ & 25 & $M$ & Tricuspid atresia & Fontan shunt & & & Amiodarone & Sinus rhythm \\
\hline & & & $\begin{array}{l}\text { Univentricular } \\
\text { heart, tricuspid } \\
\text { atresia }\end{array}$ & $\begin{array}{l}\text { Fontan (age 20), } \\
\text { closure VA leak } \\
\text { (age 28) }\end{array}$ & Calcified RA clot & $\begin{array}{r}\text { Recurrent AFl } \\
\text { since 2nd op }\end{array}$ & $\begin{array}{l}\text { Quinidine, digoxin, } \\
\text { disopyramide }\end{array}$ & $\begin{array}{l}\text { Sinus bradycardia } \\
\text { treated with } \\
\text { xamoterol. AFl } \\
\text { recurred after } 2 \\
\text { months-repeat } \\
\text { conversion to } \\
\text { sinus rhythm }\end{array}$ \\
\hline 14 & 24 & $M$ & Tetralogy of Fallot & Total repair (age 8) & $\begin{array}{l}\text { Pulmonary regurg, } \\
\text { pulm stenosis }\end{array}$ & $\begin{array}{l}\text { Recurrent AFl } \\
\text { since age } 18\end{array}$ & Amiodarone & Sinus rhythm \\
\hline 15 & 43 & $\mathbf{F}$ & Pulmonary stenosis & $\begin{array}{l}\text { Pulmonary } \\
\text { valvotomy (age 7) } \\
\text { open pulmonary } \\
\text { valvotomy + PFO } \\
\text { closure (age 16) }\end{array}$ & $\begin{array}{l}\text { Infundibular } \\
\text { hypertrophy }\end{array}$ & $\begin{array}{l}\text { Recurrent AFl } \\
\text { since age } 41\end{array}$ & $\begin{array}{l}\text { Digoxin, flecainide, } \\
\text { verapamil }\end{array}$ & Sinus rhythm \\
\hline 16 & 33 & $\mathbf{F}$ & ASD & ASD repair & Pulmonary stenosis & & Digoxin & Sinus rhythm \\
\hline
\end{tabular}
AFl, atrial flutter; ASD, atrial septal defect; AVNRT, atrioventricular nodal reentrant tachycardia; LAD, left anterior descending coronary artery; LV, left
ventricle; PA, pulmonary artery; PDA, patent ductus arteriosus; PFO, patent foramen ovale; RA, right atrium; SN, sinus node; SR, sinus rhythm; SVT, ventricle; PA, pulmonary artery; PDA, patent ductus arteriosus; PFO, patent foramen ovale; RA, right atrium; SN, sinus node; SR, sinus rhy
supraventricular tachycardia; TGA, transposition of great arteries; VA, ventriculoatrial; VSD, ventricular septal defect; VT, ventricular tachycardia.

evidence of regular atrial flutter on the surface electrocardiogram. Irregular atrial activity or atrial fibrillation was excluded. In 16 episodes, flutter was of the "common" form. In five episodes, flutter was classed as "uncommon".

\section{Results}

Table 2 shows the data gathered at electrophysiological study. All stimulation sites were in the right atrium. There was a type I response to atrial stimulation site in 16 episodes $(81 \%)$ at 24 atrial sites. No response to attempted conversion was found at eight sites (mid-lateral 1 , low lateral 1 , high anterior 2 , mid-anterior 2 , mid-septal 1, mid-posterior, 1). The distribution of the 16 type I sites at which conversion occurred was: high lateral 3 , mid lateral 2 , low lateral 2, high anterior 2, mid-anterior 3, low anterior 1 , mid-septal 1 , high posterior 1 , midposterior 1 . In addition, three type II sites were found in these 17 episodes (mid-septal 1, low septal 1, high anterior 1). Conversion was not attempted at these latter sites.

Only type II responses were found at nine sites in five episodes. Conversion did not occur at four sites (mid-lateral 1, high anterior 1, 
Table 2 Electrophysiological data on individual episodes

\begin{tabular}{|c|c|c|c|c|c|c|c|c|}
\hline Case & Age & Sex & Current treatment & $\begin{array}{l}\text { Duration } \\
\text { of flutter }\end{array}$ & Flutter type & $\begin{array}{l}\text { Atrial } \\
\text { rate }\end{array}$ & $\begin{array}{l}\text { Ventricular } \\
\text { rate }\end{array}$ & $\begin{array}{l}\text { Stimulation } \\
\text { site in } R A\end{array}$ \\
\hline $\begin{array}{r}1 \\
2 \\
3 \\
4 \\
5 \\
6 \\
7 \\
8 \\
9 \\
10 \\
11 \\
12 \\
13 \\
14 \\
15 \\
16 \\
17 \\
18 \\
19 \\
20 \\
21\end{array}$ & $\begin{array}{l}17 \\
18 \\
33 \\
20 \\
15 \\
31 \\
22 \\
34 \\
22 \\
28 \\
31 \\
22 \\
31 \\
25 \\
34 \\
31 \\
19 \\
31 \\
24 \\
43 \\
33\end{array}$ & $\begin{array}{l}M \\
M \\
F \\
F \\
M \\
F \\
F \\
M \\
F \\
M \\
M \\
F \\
M \\
M \\
M \\
M \\
F \\
M \\
M \\
\text { F } \\
\text { F }\end{array}$ & $\begin{array}{l}\text { Quinidine } \\
\text { Amiodarone } \\
\text { Nil } \\
\text { Nil } \\
\text { Amiodarone } \\
\text { Digoxin + quinidine } \\
\text { Nil } \\
\text { Nil } \\
\text { Digoxin + amiodarone } \\
\text { Amiodarone } \\
\text { Amiodarone } \\
\text { Amiodarone } \\
\text { Digoxin + quinidine } \\
\text { Amiodarone } \\
\text { Digoxin + disopyramide } \\
\text { Digoxin + disopyramide } \\
\text { Digoxin } \\
\text { Xamoterol } \\
\text { Amiodarone } \\
\text { Digoxin + flecainide } \\
\text { Digoxin }\end{array}$ & $\begin{array}{l}2 \text { days } \\
1 \text { day } \\
3 \mathrm{wk} \\
5 \mathrm{yr} \\
8 \mathrm{~h} \\
2 \text { mnth } \\
\text { Unknown } \\
3 \text { mnth } \\
\text { Unknown } \\
18 \text { mnth } \\
4 \text { days } \\
2 \text { days } \\
2 \text { wk } \\
\text { Unknown } \\
7 \text { days } \\
7 \text { days } \\
45 \text { days } \\
14 \text { days } \\
3 \text { mnth } \\
4 \text { mnth } \\
6 \text { mnth }\end{array}$ & $\begin{array}{l}\text { Common } \\
\text { Common } \\
\text { Common } \\
\text { Common } \\
\text { Uncommon } \\
\text { Uncommon } \\
\text { Uncommon } \\
\text { Common } \\
\text { Common } \\
\text { Common } \\
\text { Common } \\
\text { Common } \\
\text { Common } \\
\text { Common } \\
\text { Common } \\
\text { Common } \\
\text { Uncommon } \\
\text { Common } \\
\text { Common } \\
\text { Common } \\
\text { Uncommon }\end{array}$ & $\begin{array}{l}304 \\
221 \\
271 \\
218 \\
181 \\
363 \\
206 \\
252 \\
166 \\
219 \\
176 \\
300 \\
206 \\
172 \\
237 \\
188 \\
263 \\
330 \\
158 \\
233 \\
256\end{array}$ & $\begin{array}{r}152 \\
110 \\
135 \\
109 \\
80 \\
110 \\
103 \\
126 \\
83 \\
109 \\
88 \\
150 \\
75 \\
172 \\
79 \\
63 \\
98 \\
110 \\
79 \\
82 \\
128\end{array}$ & $\begin{array}{l}\text { Mid-lateral } \\
\text { Mid-lateral } \\
\text { High lateral } \\
\text { Low lateral } \\
\text { High anterior } \\
\text { Low lateral } \\
\text { Mid lateral } \\
\text { Low septal } \\
\text { Low posterior } \\
\text { Mid posterior } \\
\text { High posterior } \\
\text { Low lateral } \\
\text { High lateral } \\
\text { High anterior } \\
\text { Mid-anterior } \\
\text { Mid-anterior } \\
\text { High anterior } \\
\text { Mid-anterior } \\
\text { High anterior } \\
\text { Low anterior } \\
\text { High lateral }\end{array}$ \\
\hline
\end{tabular}

mid-anterior 1, high septal 1). Conversion occurred at the remaining five sites (mid-lateral 1 , low lateral 1 , high anterior 2 , low posterior 1).

Single atrial stimuli did not convert any episode of flutter. Conversion to sinus rhythm was achieved with programmed stimuli in six episodes. Figure 2 shows an example of conversion with two stimuli. Rapid atrial pacing resulted in conversion to sinus rhythm in 11 episodes (figure 3 shows one) and to atrial fibrillation in two. One of these latter two episodes had the most rapid atrial rate (363 beats per minute) and endocardial mapping showed coexisting areas of atrial fibrillation and flutter not suspected from the surface electrocardiogram; sinus rhythm spontaneously resumed 12 hours later. In the other episode where atrial fibrillation ensued, DC cardioversion was carried out immediately because the patient had documented sinus node disease and the atrial catheter would have allowed backup atrial pacing if required.

The remaining two episodes in one patient were converted to sinus rhythm by a single long constant current pulse ("atrioversion"8). Fifteen of the 19 conversions to sinus rhythm were at type I atrial sites.
One conversion to sinus rhythm after rapid atrial pacing was followed by profound, sustained sinus bradycardia, requiring atrial support pacing and subsequently isoprenaline infusion to maintain an adequate rate. The patient was subsequently treated with oral xamoterol, but had a further recurrence of atrial flutter two months later and was again successfully converted to sinus rhythm. Xamoterol was stopped and an atrial demand pacemaker was implanted.

Overall, 19 episodes $(90 \%)$ were returned directly to sinus rhythm during the procedure. One patient, who had coexisting areas of atrial fibrillation not suspected from the surface electrocardiogram and was converted to atrial fibrillation, subsequently reverted to sinus rhythm after 12 hours. Clinical success was thus achieved in 20 episodes (95\%). In all patients the clinical state improved.

\section{Discussion}

In patients with one functioning ventricle, as in tricuspid atresia or double inlet ventricle, the onset of atrial flutter causes a considerable symptomatic deterioration. Atrial flutter is also dangerous in other complex lesions, such as
Figure 2 Conversion of atrial flutter to sinus rhythm by two premature atrial stimuli. Four surface electrocardiographic leads and two intracardiac electrograms showing atrial flutter with a cycle length of $220 \mathrm{~ms}$.

Two extrastimuli

(coupling times $140 \mathrm{~ms}$ and $180 \mathrm{~ms}$ ) caused immediate reversion to sinus rhythm.

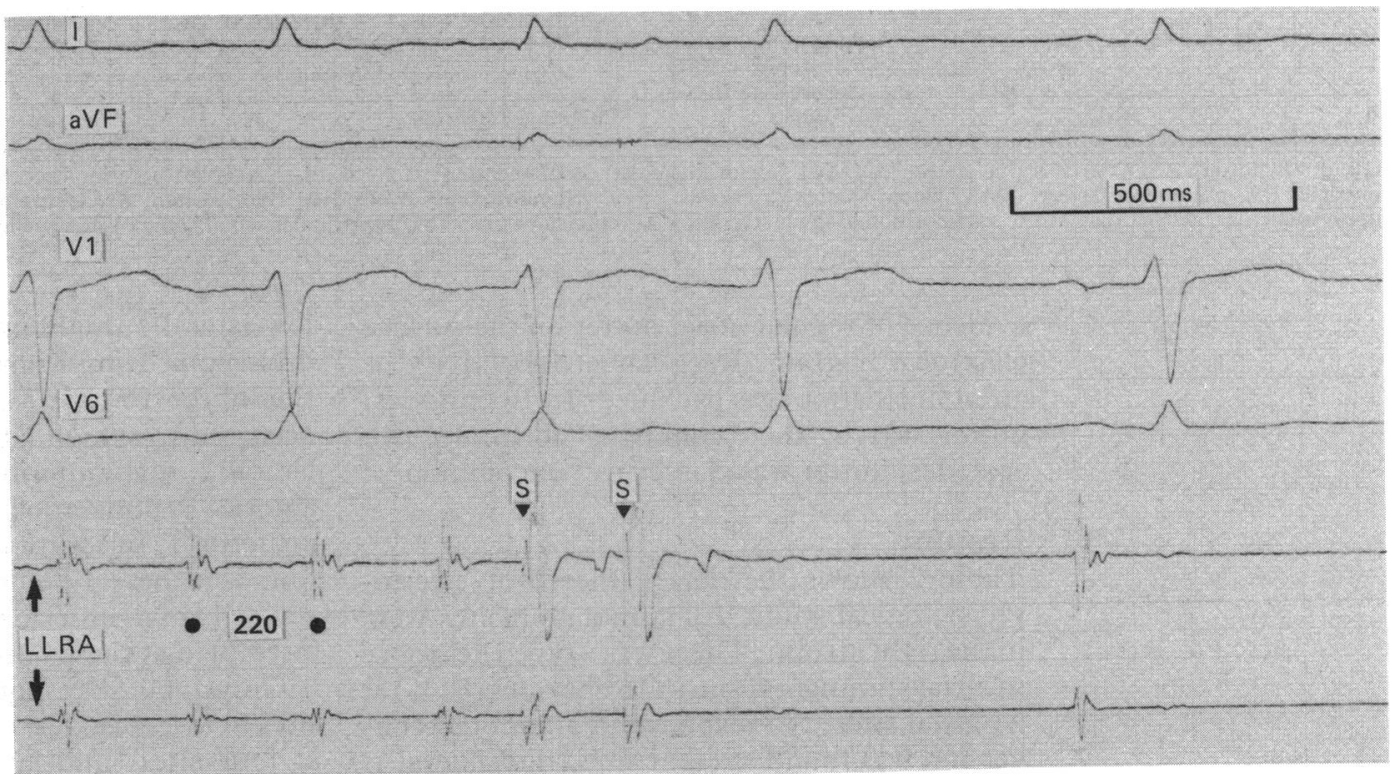




\begin{tabular}{|c|c|c|c|}
\hline Response type & Conversion method & Result of conversion & $\begin{array}{l}\text { Details of } \\
\text { conversion }\end{array}$ \\
\hline $\begin{array}{l}\text { Type I (reset) } \\
\text { Type I (reset) } \\
\text { Type I (reset) } \\
\text { Type I (reset) } \\
\text { Type II (non-reset) } \\
\text { Type II (non-reset) } \\
\text { Type II (non-reset) } \\
\text { Type I (reset) } \\
\text { Type I (reset) } \\
\text { Type I (reset) } \\
\text { Type I (reset) } \\
\text { Type I (reset) } \\
\text { Type I (reset) } \\
\text { Type II (non-reset) } \\
\text { Type I (reset) } \\
\text { Type I (reset) } \\
\text { Type I (reset) } \\
\text { Type I (reset) } \\
\text { Type I (reset) } \\
\text { Type I (reset) } \\
\text { Type I (reset) }\end{array}$ & $\begin{array}{l}\text { Atrioversion } \\
\text { Atrioversion } \\
\text { Extrastimuli } \\
\text { Extrastimuli } \\
\text { Rapid atrial pacing } \\
\text { Rapid atrial pacing } \\
\text { Extrastimuli } \\
\text { Extrastimuli } \\
\text { Extrastimuli } \\
\text { Rapid atrial pacing } \\
\text { Extrastimuli } \\
\text { Rapid atrial pacing } \\
\text { Rapid atrial pacing } \\
\text { Rapid atrial pacing } \\
\text { Rapid atrial pacing } \\
\text { Rapid atrial pacing } \\
\text { Rapid atrial pacing } \\
\text { Rapid atrial pacing } \\
\text { Rapid atrial pacing } \\
\text { Rapid atrial pacing } \\
\text { Rapid atrial pacing }\end{array}$ & $\begin{array}{l}\text { Sinus rhythm } \\
\text { Sinus rhythm } \\
\text { Sinus rhythm } \\
\text { Sinus rhythm } \\
\text { Sinus rhythm } \\
\text { Atrial fibrillation } \\
\text { Sinus rhythm } \\
\text { Sinus rhythm } \\
\text { Sinus rhythm } \\
\text { Sinus rhythm } \\
\text { Sinus rhythm } \\
\text { Sinus rhythm } \\
\text { Sinus rhythm } \\
\text { Sinus rhythm } \\
\text { Sinus rhythm } \\
\text { Sinus rhythm } \\
\text { Atrial fibrillation } \\
\text { Sinus rhythm } \\
\text { Sinus rhythm } \\
\text { Sinus rhythm } \\
\text { Sinus rhythm }\end{array}$ & $\begin{array}{l}180 / 170 \mathrm{~ms} \\
170 / 140 \mathrm{~ms} \\
\text { CL } 165 \mathrm{~ms} \text { for } 4 \mathrm{~s} \\
\text { CL } 100 \mathrm{~ms} \text { for } 8 \mathrm{~s} \\
270 / 250 \mathrm{~ms} \\
155 / 140 / 140 / 140 \mathrm{~ms} \\
270 / 210 \mathrm{~ms} \\
\text { CL } 200 \mathrm{~ms} \text { for } 4 \mathrm{~s} \\
240 / 240 / 290 \mathrm{~ms} \\
\text { CL } 210 \mathrm{~ms} \text { for } 8 \mathrm{~s} \\
\text { CL } 200 \mathrm{~ms} \text { for } 4 \mathrm{~s} \\
\text { CL } 290 \mathrm{~ms} \text { for } 8 \mathrm{~s} \\
\text { CL } 160 \mathrm{~ms} \text { for } 4 \mathrm{~s} \\
\text { CL } 230 \mathrm{~ms} \text { for } 30 \mathrm{~s} \\
\text { CL } 160 \mathrm{~ms} \text { for } 4 \mathrm{~s} \\
\text { CL } 150 \mathrm{~ms} \text { for } 8 \mathrm{~s} \\
\text { CL } 300 \mathrm{~ms} \text { for } 2 \mathrm{~s} \\
\text { CL } 170 \mathrm{~ms} \text { for } 4 \mathrm{~s} \\
\text { CL } 180 \mathrm{~ms} \text { for } 2 \mathrm{~s}\end{array}$ \\
\hline
\end{tabular}

CL, cycle length. coexisting areas of flutter and fibrillation that were not evident on the surface electrocardiogram. Conversion to sinus rhythm was thus unlikely in this episode.

For this study we selected patients with regular and rapid atrial arrhythmia. Patients with anomalies of systemic venous drainage were excluded owing to difficulties of access. Otherwise, it is our policy to start anticoagulation with heparin and to proceed to electrical conversion as soon as is possible. Appropriate antiarrhythmic medication to prevent recurrences is instituted or continued after conversion. We advise patients to return immediately if a further paroxysm occurs.

In the five instances in which the atrial rate was below 200 beats per minute during flutter, concomitant drug treatment accounted for the relatively slow atrial rate. A gradual lengthening of the flutter cycle without a change in flutter wave configuration was seen on sequential electrocardiographic recordings after treatment was started. Because this effect was consistent with the electrophysiological effect of amiodarone and disopyramide we thought that the mechanism of these slower rhythms must be identical and that they should remain classified as atrial flutter.

As well as having a high success rate, endocardial paced conversion of atrial flutter allows immediate atrial support pacing when there is sinus arrest after conversion-a facility not available during external cardioversion. This proved essential in one episode where profound sinus bradycardia followed conversion.

All of the study group had had previous corrective surgery, usually at an early age. None had a history of atrial arrhythmias before operation, and it is probable that the surgery contributed in a major way to creation of the substrate for their atrial flutter.

The procedure was highly successful in this group of patients. The mean procedure time
Figure 3 Conversion of atrial flutter to sinus rhythm by rapid atrial overdrive pacing. Four surface electrocardiographic leads and two intracardiac electrograms from the low lateral right atrium ( $L L R A$ ) show the last four beats of an $8 \mathrm{~s}$ overdrive.pacing train (cycle length $210 \mathrm{~ms}$ ). After the end of pacing there were two atrial depolarisations, the first of which may be sinus in origin, followed by clear resumption of sinus rhythm.

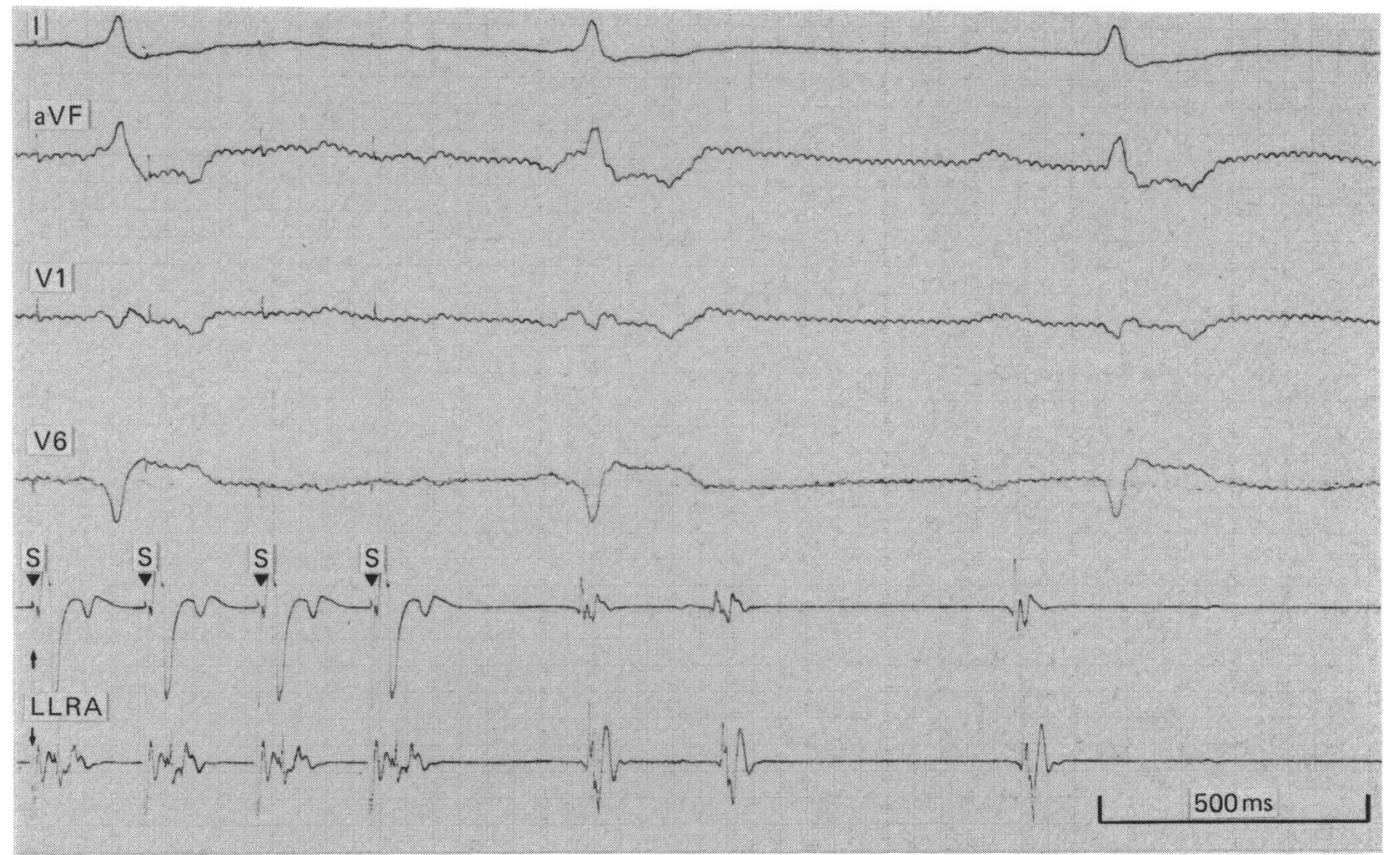


was an hour, and theoretically the procedure could be carried out at the bedside under echocardiographic control. We believe that intracardiac electrical conversion is the best treatment for atrial flutter in patients with congenital heart disease because it avoids general anaesthesia and external cardioversion.

1 Dahl CF, Ewy GA, Warner ED, Thomas ED. Myocardia necrosis from direct current discharge. Effect of paddle electrode size and time interval between discharges. Circulation 1974;50:956-61.

2 Davis JS, Lie JT, Bentinck DC, Titus JL, Tacker WA Geddes LA. Cardiac damage due to electrical current and energy. Proceedings of the cardiac defibrillation conference, West Lafayette, Indiana. Purdue University,
3 Wilson CM, Allen JD, Bridges JB, Adgey AAJ. Death and damage caused by multiple direct current shocks: studies in an animal model. Eur Heart $J$ 1988;9:1257-65.

4 Haft JI, Kosowsky BD, Lau SH, Stein E, Damato AN. Termination of atrial flutter by rapid electrical pacing of the atrium. Am J Cardiol 1967;20:239-44.

5 Das G, Anand MK, Ankineedu K, Chinnavaso T, Talmers FN, Weissler AM. Atrial pacing for cardioversion of atrial flutter in digitalized patients. Am J Cardiol 1978;41: 308-12. Ward D, Spurrell R. Response of atrial flutter to 6 Camm J, Ward D, Spurrell R. Response of atrial flutter to overdrive atrial pacing and intravenous disopyramide
phosphate, singly and in combination. Br Heart $J$ phosphate, sing

7 Waldo AL, MacLean WAH, Karp RB, Kouchokos NT James TN. Entrainment and interruption of atrial flutter with atrial pacing: studies in man following open heart surgery. Circulation 1977;56:737-45.

8 Cunningham AD, Kennedy JA. Atrioversion-conversion of atrial flutter in man using a long constant current pulse [Abstract]. Br Heart J 1985;53:93. 